\title{
Threshold Voltage Compensation Error in Voltage Programmed AMOLED Displays
}

\author{
Mojtaba Bagheri, Student Member, IEEE, Xiang Cheng, Junhao Zhang, Sungsik Lee, Shahin Ashtiani, \\ Arokia Nathan, Fellow, IEEE
}

\begin{abstract}
A new accurate voltage-programmed pixel circuit for active matrix organic light-emitting diode (AMOLED) displays is presented. Composed of three TFTs and one storage capacitor, the proposed pixel circuit is implemented both in a-Si and a-IGZO TFT technologies for the same pixel size for fair comparison. The simulation result for the a-Si-based design shows that, during a programming time of $90 \mu \mathrm{s}$, the pixel circuit was able to compensate for a $3 \mathrm{~V}$ threshold voltage $\left(V_{\text {th }}\right)$ shift of the drive TFT with almost no error. In contrast, the a-IGZO-based pixel circuit, has a larger current error (of around $8 \%$ ), despite its proven three-fold higher speed.
\end{abstract}

Index Terms-active-matrix organic light-emitting diode (AMOLED), amorphous silicon (a-Si), oxide thin-film transistor (TFT), compensation.

\section{INTRODUCTION}

$\mathbf{O}$ WNING to their competitive advantages over the ubiquitous liquid crystal display (LCD), organic light-emitting diode (OLED) displays, integrated with thin film transistor (TFT) technology, have generated considerable interest in recent years. The process of compensation for the threshold voltage shift as an intrinsic property of the TFT, differentiates the driving scheme of active matrix OLED (AMOLED) display from its LCD counterpart.

Among various technologies to implement TFT backplanes, there is amorphous silicon (a-Si), low-temperature polycrystalline silicon (LTPS) and amorphous indium gallium zinc oxide (a-IGZO). LTPS offers higher mobility and generally lower parasitic capacitance compared to IGZO depending on device structure [1]. However, it suffers from short range mismatch due to grain boundaries. The fabrication process is more costly especially when it comes to large area scaling due to the more complex processing. IGZO technology, which belongs to the general category of metal-oxide semiconductor, offers a carrier mobility of at least 15 times higher compared to the silicon-based technology [2]. This and the low temperature fabrication process ${ }^{1}$ (enabling flexible displays) as well as higher stability of threshold voltage shift under positive gatebias stress [3] have made this new technology very attractive for implementing circuits, including image capture [4], in AMOLED displays.

It is well known that the threshold voltage shift has a direct impact on ciruit performance. For example, consider the simple 2-TFT pixel circuit shown in Fig. 1. The data line provides the required programming voltage for the drive TFT,

${ }^{1}$ Mainly because the deposition method that is used for fabricating a-IGZO TFTs is Physical Vapour Deposition (PVD) rather than Chemical Vapour Deposition (CVD).

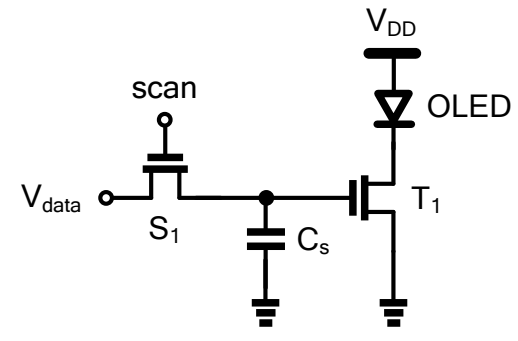

Fig. 1: A simple structure for AMOLED pixel.

while the scan line determines the running state of the switch TFT, i.e. ON or OFF. The voltage stored on $C_{s}$ is converted to a current by $T_{1}$, which passes through the OLED. Due to the voltage shift in $V_{\text {th }}$ of $T_{1}$, this simple circuit cannot be used as a practical pixel configuration to drive the OLED, because the current and thus the luminance of the OLED degrades for a specific data voltage over time. Since this shifting process of the threshold voltage of a TFT under gate-source stress is not accurately predictable, and circuit designers have been persuaded to propose diverse techniques to compensate for the aforementioned instability of the AMOLED pixel circuits and stabilize the OLED luminance [5].

Among the different methods proposed for $V_{\text {th }}$ compensation, the voltage-programming based drive scheme [5-9] has attracted considerable attention in view of its advantages such as faster settling time. In essence, in all voltage-programming schemes, a storage capacitor $\left(C_{s}\right)$ is precharged to a desired voltage, and during the compensation period, it discharges through a diode-connected TFT (drive TFT, $T_{1}$ ) until its voltage reaches the threshold voltage, as illustrated in Fig. 2. At this time, $T_{1}$ goes OFF and $C_{s}$ stops discharging. After that, the data voltage ( $\left.V_{\text {data }}\right)$ is added to the voltage across $C_{s}$, making the gate-source voltage of $T_{1} V_{\text {data }}+V_{\text {th }}$. Supposing $T_{1}$ is in the saturation region, the current through $T_{1}$ would be independent of $V_{\text {th }}$ and is given by

$$
\begin{aligned}
I_{\mathrm{OLED}} & =\frac{1}{2} K\left(V_{g}-V_{\mathrm{th}}\right)^{2} \\
& =\frac{1}{2} K\left(V_{\mathrm{data}}+V_{\mathrm{th}}-V_{\mathrm{th}}\right)^{2} \\
& =\frac{1}{2} K V_{\mathrm{data}}^{2},
\end{aligned}
$$

where

$$
K=\mu_{\mathrm{FET}} C_{i} \frac{W}{L},
$$

and $\mu_{\mathrm{FET}}, C_{i}, W$, and $L$ are field effect mobility, gate insulator 


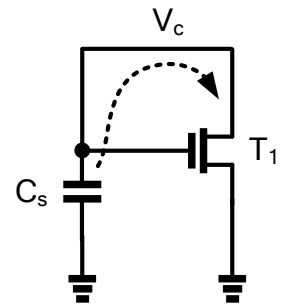

Fig. 2: Compensation phase of a voltage-programmed pixel circuit.

capacitance, channel width, and channel length, respectively. This very simple method is, however, flawed in some respects: First and foremost, the time constant of the circuit is determined by the transconductance $\left(g_{m}\right)$ of the drive TFT, the value of which depends on the voltage of the top plate of the capacitor (which is the voltage of the gate (drain) of the drive TFT). As this voltage degrades, $g_{m}$ also reduces, making the circuit very slow to reach the desired $V_{\text {th }}$. The final overdrive voltage of the drive TFT can be obtained as [10]

$$
V_{\mathrm{ov}}=\frac{V_{C 0}-V_{\mathrm{th}}}{\left(V_{C 0}-V_{\mathrm{th}}\right) \frac{K}{2 C_{s}} t_{c}+1}
$$

where $V_{C 0}$ is the initial voltage of the capacitor, and $t_{c}$ is the compensation time.

Second, even when $V_{C}$ reaches $V_{\text {th }}$, due to the subthreshold current, it still keeps decreasing, making it impossible to accurately measure the threshold voltage.

A new method of compensation was devised in [11] that yielded a fast, accurate pixel circuit. This was not however at the expense of circuit complexity. In this work, we adopt the same methodology, but with a reduced complexity circuit. The circuit has one less TFT, one less capacitor, and one less control line. An analytical description of the functionality of the compensation approach is also presented.

\section{Analysis of the Proposed $V_{\mathrm{TH}}$ Compensation METHOD}

Fig. 3 shows the concept of the new driving scheme. The circuit consists of a capacitor $\left(C_{1}\right)$ which is connected to the drain of the drive TFT $\left(T_{1}\right)$ (Fig. 3(a)). If we precharge $C_{1}$ to a voltage, say $V_{C 0}$, and apply an arbitrary voltage function to the gate of $T_{1}$ (Fig. 3(b)) for a definite time (from $t=0$ to $t=t_{1}$ ), $C_{1}$ starts discharging until the gate voltage becomes zero. Now, assume that the threshold voltage of $T_{1}$ shifts (to a more positive one). If the gate voltage function remains unchanged, $T_{1}$ experiences a smaller gate-source voltage during the discharging interval. Hence, the current which causes $C_{1}$ to discharge is now smaller, resulting in a larger final voltage stored on the top plate of $C_{1}\left(V_{C}\left(t_{1}\right)\right)$.

To summarize, we can state that, as the threshold voltage of $T_{1}$ shifts, the final voltage stored across $C_{1}$ increases. This is the principle of the proposed $V_{\text {th }}$ compensation technique.

In the proposed circuit, the applied voltage is a constant equalling $V_{g 0}$. In order to work out the final voltage of $C_{1}$ at the end of the discharging interval, we assume the initial

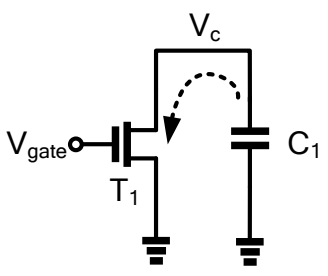

(a)

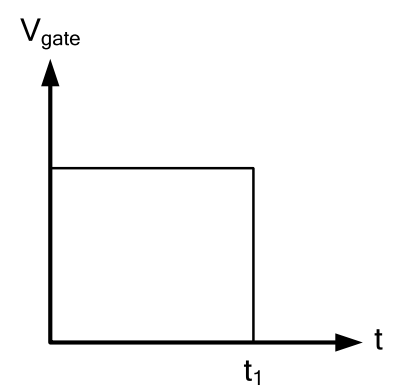

(b)

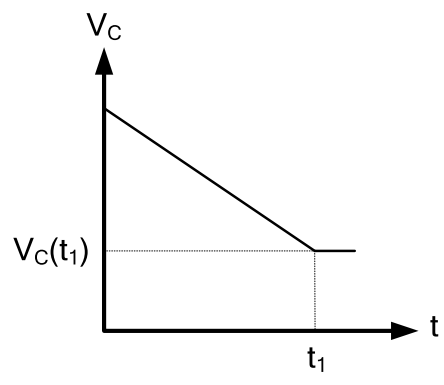

(c)
Fig. 3: (a) Circuit core that compensates for $V_{\text {th }}$ (b) applied gate voltage (c) voltage across the capacitor.

voltage of the capacitor to be $V_{C 0}$. Now, from the simple square-law characteristic of a field-effect transistor, which is

$$
I=\frac{1}{2} K\left(V_{g 0}-V_{\mathrm{th}}\right)^{2},
$$

and the current-voltage relation for a linear capacitor, which is

$$
i=C \frac{\triangle V}{\triangle t},
$$

the final voltage of $C_{1}$ is readily derived and is given by

$$
V_{C}\left(t_{1}\right)=V_{C 0}-\frac{K}{2 C_{1}}\left(V_{g 0}-V_{\mathrm{th}}\right)^{2} t_{1} \text {. }
$$

If $V_{\mathrm{th} 0}$ is the initial threshold voltage of $T_{1}$, and $\Delta V_{\mathrm{th} 0}$ is the threshold voltage shift, (6) can be rewritten as

$$
V_{C}\left(t_{1}\right)=V_{C 0}-\frac{K}{2 C_{1}}\left(V_{g 0}-V_{\mathrm{th} 0}-\Delta V_{\mathrm{th}}\right)^{2} t_{1} .
$$

Expanding the above equation, we have

$$
\begin{aligned}
V_{C}\left(t_{1}\right)= & {\left[V_{C 0}-\frac{K}{2 C_{1}}\left(V_{g 0}-V_{\mathrm{th} 0}\right)^{2} t_{1}\right] } \\
& +\frac{K t_{1}}{2 C_{1}}\left[2\left(V_{g 0}-V_{\mathrm{th} 0}\right) \Delta V_{\mathrm{th}}-\Delta V_{\mathrm{th}}^{2}\right] .
\end{aligned}
$$

The first group of terms in (8) is independent of $\Delta V_{\text {th }}$. We name it $V_{\text {ind }}$. Following (8), and by adding the data voltage, $V_{\text {data }}$, to $V_{C}\left(t_{1}\right)$, the current through the OLED is then given by

$$
\begin{aligned}
I_{\mathrm{OLED}} & =\frac{1}{2} K\left(V_{\mathrm{data}}+V_{\mathrm{ind}}\right. \\
& \left.+\frac{K t_{1}}{2 C_{1}}\left[2\left(V_{g 0}-V_{\mathrm{th} 0}\right) \Delta V_{\mathrm{th}}-\Delta V_{\mathrm{th}}^{2}\right]-V_{\mathrm{th}}\right)^{2} \\
& =\frac{1}{2} K\left(V_{\mathrm{data}}+V_{\mathrm{ind}}-V_{\mathrm{th} 0}\right. \\
& \left.+\frac{K t_{1}}{2 C_{1}}\left[2\left(V_{g 0}-V_{\mathrm{th} 0}\right) \Delta V_{\mathrm{th}}-\Delta V_{\mathrm{th}}^{2}\right]-\Delta V_{\mathrm{th}}\right)^{2}
\end{aligned}
$$




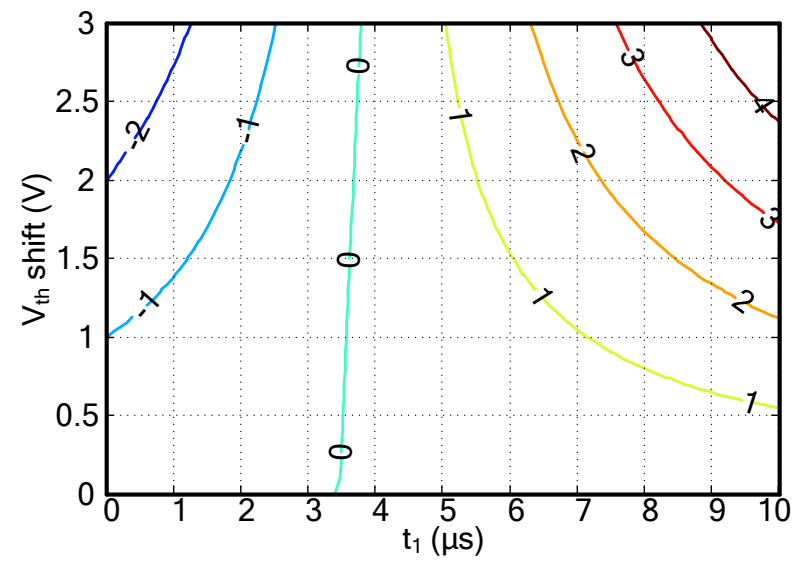

Fig. 4: The profile of the error voltage during OLED compensation in the proposed pixel circuit.

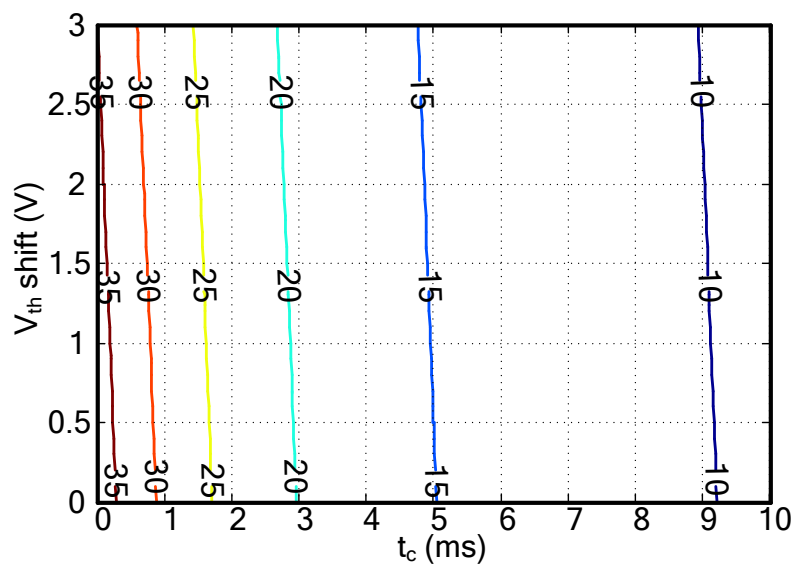

Fig. 5: The profile of the error voltage during OLED compensation in the conventional pixel circuit.

The $V_{\text {th }}$-dependent terms in (9) need to be minimized within a defined range of $V_{\text {th }}$ shift to reach an optimum point for the current error of the OLED. Typical values for $\mathrm{K}$ and $V_{\text {tho }}$ for an a-Si TFT with $W / L=10 \mu m / 10 \mu m$ (used in the simulations) are close to $16 n A / V^{2}$ and $2 V$, respectively. The optimization is conducted for $0<\Delta V_{\text {th }}<3 V$ and $C_{1}$ and $V_{g 0}$ are chosen as $1 p F$ and $20 \mathrm{~V}$, respectively. The profile of the $V_{\mathrm{th}}-$ dependent terms (error voltage), i.e.

$$
f\left(t_{1}, \Delta V_{\mathrm{th}}\right)=\frac{K t_{1}}{2 C_{1}}\left[2\left(V_{g 0}-V_{\mathrm{th} 0}\right) \Delta V_{\mathrm{th}}-\Delta V_{\mathrm{th}}^{2}\right]-\Delta V_{\mathrm{th}},
$$

is numerically analysed and plotted in Fig. 4. As can be seen, the error voltage is approximately zero around $t_{1}=3.5 \mu \mathrm{s}$. For sake of comparison, the error voltage in (3), which is the second term, is also plotted in Fig. 5. A similar simulation demonstrates that in order to reach an error comparable with that of the proposed circuit, $t_{c}$ must be in the order of $1 \mathrm{~ms}$, while the maximum programming time budget is around $70 \mu \mathrm{s}$ in QVGA displays and less than $45 \mu$ s in XVGA ones [10].

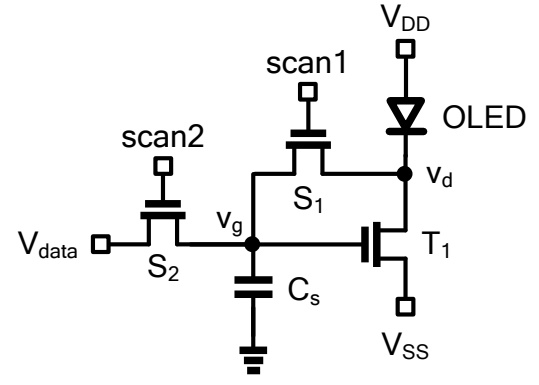

Fig. 6: Proposed pixel circuit for $V_{\text {th }}$ compensation.

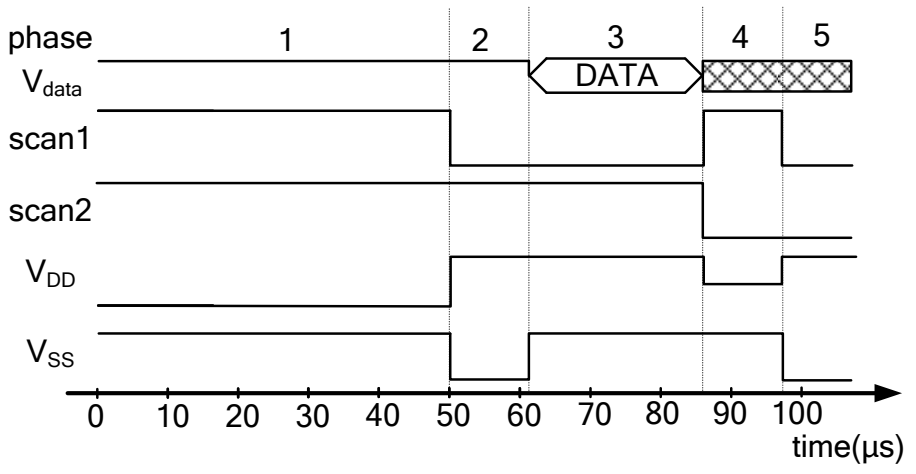

Fig. 7: Timing diagram for the various lines for the a-Si circuit.

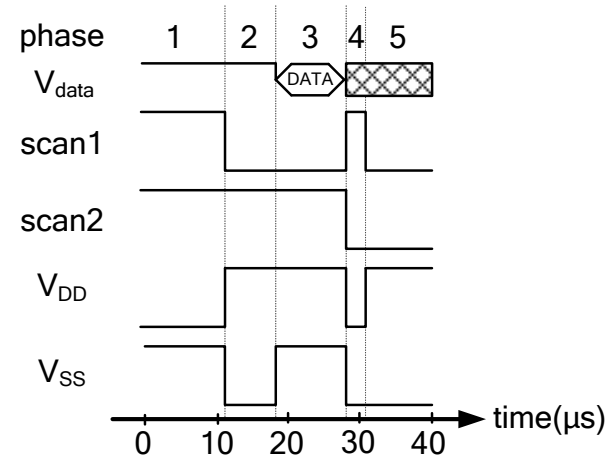

Fig. 8: Timing diagram for the various lines for the a-IGZO circuit.

\section{Circuit Implementation In a-Si and a-IGZO Technologies}

In this section, the programming process of the proposed pixel circuit is analysed in two thin film technlogies: a-Si TFT and a-IGZO TFT. To have a fair comparison, we should assume the same size of the pixel for both implementations. This in particular means equal sizes of the drive TFT, the switches and the storage as well as the OLED capacitor. Fig. 6 shows the structure of the circuit. As can be seen, it is composed of three TFTs and one capacitor. $V_{\text {data }}$ provides the data voltage, and $V_{\mathrm{DD}}, V_{\mathrm{SS}}$, scan1, and scan2 are the controlling lines. Fig. 7 and Fig. 8 demonstrate the driving sequence of the pixel using a-Si and a-IGZO TFTs, respectively. The driving sequence divided into five main phases which will be elaborated in the following. 


\section{A. Driving Sequence}

In the first phase, i.e. the initialization phase, scan1, scan2 and $V_{\mathrm{DD}}$ are high, high, and low, respectively. $V_{\mathrm{SS}}$ is also high, making $T_{1}$ OFF. Since the voltage of the cathode in the OLED is greater than that of the anode, the OLED is reversed-bias and acts as a capacitor $C_{\mathrm{OLED}}{ }^{2}$, similar to $C_{1}$ in Fig. 3. At this time, $V_{\text {data }}$ is set to a constant voltage (initializing), precharging $v_{d}$ and the top plate of $C_{s}$ through the two switches $S_{1}$ and $S_{2}$.

In the second phase, i.e. the compensation phase, scan 1 and scan2 are low and high respectively. $V_{\mathrm{DD}}$ turns back to its maximum, introducing a jump on node $v_{d}$ via the floating OLED capacitor $C_{\text {OLED }}$. This causes $v_{d}$ to reach a relatively high voltage. While $V_{\text {data }}$ remains unchanged ${ }^{3}, V_{\mathrm{SS}}$ is pulled down, turning the drive TFT $T_{1}$ ON. As a result of that, $C_{\text {OLED }}$ starts discharging through $T_{1}$ with a constant rate for a period of $t_{\text {comp }}$. Here, $t_{\text {comp }}$ should be chosen appropriately to reach a minimum error, as will be discussed below.

In the third phase, i.e. the programming phase, scan1, scan2, $V_{\mathrm{DD}}$ are low, high, and high, respectively. Pulling $V_{\mathrm{SS}}$ up, $T_{1}$ turns off and $C_{\text {OLED }}$ stops discharging. Assuming a total compensation time of $t_{\text {comp }}$ and according to (8), the final voltage of the bottom plate of $C_{\mathrm{OLED}}\left(v_{d}\right)$ can be written as

$$
V_{d}=V_{\text {ind }}+\frac{K t_{\text {comp }}}{2 C_{\text {OLED }}}\left[2\left(V_{\text {data2 }}-V_{\text {th0 } 0}\right) \Delta V_{\text {th }}-\Delta V_{\text {th }}^{2}\right],
$$

where $V_{g 0}$ in (9) is replaced by $V_{\text {data2 }}$, and represents the value of $V_{\text {data }}$ in the second phase.

Simultaneously, the data voltage $\left(V_{\text {data }}\right)$ is also applied to the top plate of $C_{s}$ via $S_{2}$.

The fourth phase, i.e. the charge sharing phase, starts with pulling scan1 up and scan2 down. Doing so, the bottom plate of $C_{\mathrm{OLED}}$ connects to the top plate of $C_{s}$ and a charge sharing occurs. The final voltage of the top plates after settling depends on the ratio of the two capacitors. Assuming a ratio of $C_{s} / C_{\mathrm{OLED}}=\alpha$ and according to (11), this voltage would be

$$
\begin{aligned}
V_{g}= & \frac{\alpha}{\alpha+1} V_{\text {data }} \\
& +\frac{1}{\alpha+1}\left(V_{\text {ind }}+\frac{K t_{\text {comp }}}{2 C_{\text {OLED }}}\left[2\left(V_{\text {data2 }}-V_{\text {th } 0}\right) \Delta V_{\text {th }}-\Delta V_{\text {th }}^{2}\right]\right) .
\end{aligned}
$$

At this time, since the overall voltage across $C_{\mathrm{OLED}}$ decreases (especially for smaller $V_{\text {data }} \mathrm{s}$ ), the OLED may enter the forward-bias regime and it no longer acts as a capacitor. To avoid this to happen, we reduce $V_{\mathrm{DD}}$ in the beginning of the phase.

In the final phase, i.e. the driving phase, scan1 and scan2 are low, and $V_{\mathrm{DD}}$ returns to its default value, and $V_{\mathrm{SS}}$ is pulled down. A current proportional to the voltage of $C_{s}$, which is

${ }^{2}$ From [12] a typical value for this capacitance is $200-400 \mathrm{pF} / \mathrm{mm}^{2}$. An area of $500 \mu \mathrm{m}^{2}$ for the OLED would result to an OLED capacitor of $1 p F$.

${ }^{3} V_{\text {data }}$ can change here to a different value of that in phase 1 , but, for whatever $V_{\text {data }}$ in either phases, one should make sure that the drive transistor $T_{1}$ always remains in the saturation region, i.e. $V_{D 1}>V_{G 1}-V_{\text {th1 }}$, where $V_{D 1}, V_{G 1}$ and $V_{\text {th1 }}$ are the drain voltage, the gate voltage and the threshold voltage of $T_{1}$. given by (12), passes through the OLED, i.e,

$$
\begin{aligned}
I_{\mathrm{OLED}} & =\frac{1}{2} K\left(V_{g}-V_{\mathrm{th}}\right)^{2} \\
& =\frac{1}{2} K\left(\frac{\alpha}{1+\alpha} V_{\mathrm{data}}+\frac{1}{1+\alpha}\left(V_{\mathrm{ind}}+\frac{K t_{\mathrm{comp}}}{2 C_{\mathrm{OLED}}}\right.\right. \\
& {\left.\left.\left[2\left(V_{\mathrm{data} 2}-V_{\mathrm{th} 0}\right) \Delta V_{\mathrm{th}}-\Delta V_{\mathrm{th}}^{2}\right]\right)-V_{\mathrm{th}}\right)^{2} } \\
& =\frac{1}{2} K\left(\frac{\alpha}{1+\alpha} V_{\mathrm{data}}+\frac{1}{1+\alpha}\left(V_{\mathrm{ind}}+\frac{K t_{\mathrm{comp}}}{2 C_{\mathrm{OLED}}}\right.\right. \\
& {\left.\left.\left[2\left(V_{\mathrm{data} 2}-V_{\mathrm{th} 0}\right) \Delta V_{\mathrm{th}}-\Delta V_{\mathrm{th}}^{2}\right]\right)-\Delta V_{\mathrm{th}}-V_{\mathrm{th} 0}\right)^{2} . }
\end{aligned}
$$

As can be seen, there is an undesirable $V_{\text {th }}$-dependent term (the error) in (11), which is

$$
\begin{aligned}
V_{\text {error }} & = \\
& \quad \frac{1}{1+\alpha}\left(\frac{K t_{\text {comp }}}{2 C_{\text {oLED }}}\left[2\left(V_{\text {data2 }}-V_{\text {th0 }}\right) \Delta V_{\text {th }}-\Delta V_{\text {th }}^{2}\right]\right) \\
& -\Delta V_{\text {th }} .
\end{aligned}
$$

This is a parabolic curve with respect to $\Delta V_{\text {th }}$. Assuming $0<$ $\Delta V_{\text {th }}<\Delta V_{\text {th,max }}$, the maximum (absolute) voltage error occurs either at the peak (vertex) of the curve or at the edge of the definition range (where $\Delta V_{\mathrm{th}}=\Delta V_{\mathrm{th} \text { max }}$ ). To reach a minima for the error, the larger one of the two should be minimized, and this proves to be where the error values at these two points are equal. Based on this, we derive the optimum value for the compensation time as

$$
t_{\text {comp,opt }}=\frac{C_{s}+C_{O L E D}}{K\left[V_{\text {data2 }}-V_{\text {th0 }}-(\sqrt{2}-1) \Delta V_{\text {th,max }}\right]}
$$

and the maximum voltage error as

$$
V_{\text {error,max }}=\frac{(3-2 \sqrt{2}) \Delta V_{\mathrm{th}, \text { max }}^{2}}{2\left[V_{\mathrm{data} 2}-V_{\mathrm{th} 0}-(\sqrt{2}-1) \Delta V_{\mathrm{th}, \max }\right]}
$$

Therefore, the error is totally determined by the gate voltage during the second phase $V_{\text {data2 }}$ (or equivalently the overdrive voltage $V_{o v}=V_{\text {data2 }}-V_{\text {th }}$ ) and the maximum $V_{\text {th }}$ shift $\left(\Delta V_{\text {th,max }}\right)$. For $V_{\text {th0 }}=2 V$, this maximum error is plotted in Fig. (9) as a function of the overdrive voltage during the compensation phase. As can be seen, for large overdrive voltages, the error is very small and negligible.

We can use (15) to calculate an approximation ${ }^{4}$ for $t_{\text {comp }}$. We can alternatively perform the procedure in section II (and related to Fig. 4) to calculate the optimum compensation time. The most accurate method, however, is to use a trial-anderror approach through simulation with the real circuit models. Using the extracted model parameters of an a-Si TFT and for an OLED capacitor of $C_{\mathrm{OLED}}=1 p F$ and $\alpha$ between 0.5 and 1 , this optimum time as well as the corresponding current error is obtained and plotted in Fig. 10. The same trend can be followed for an a-IGZO TFT.

\section{B. Driving/Circuit Discrepancies in the Two Technologies}

The phases and their driving sequence are exactly the same for both circuits implemented in a-Si and a-IGZO technologies. As such, if we simply get the signaling of the circuit in

\footnotetext{
${ }^{4}$ This is indeed an apprpximate value because all the secondary effects, e.g. the channel length modulation, as well as the junction and parasitic capacitances of the drive TFT are neglected.
} 


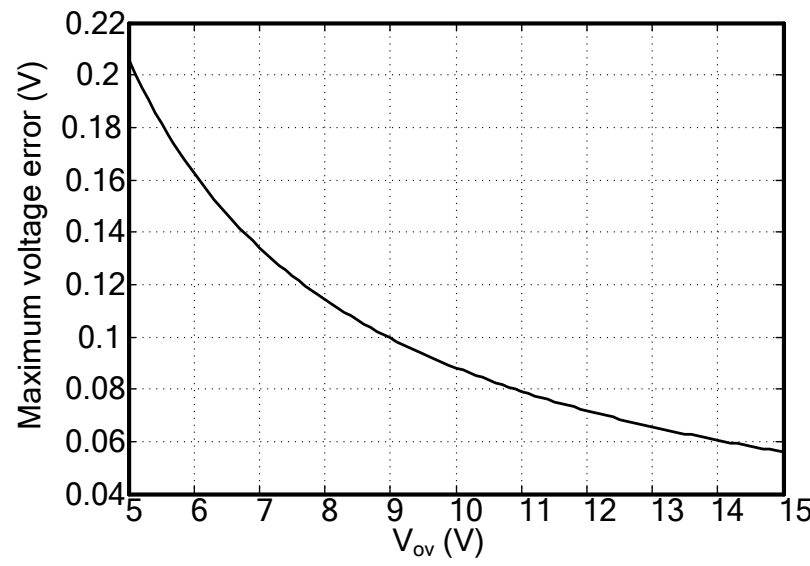

Fig. 9: Maximum voltage error rate as a function of overdrive voltage during the second phase for a threshold voltage shift of $3 \mathrm{~V}$.

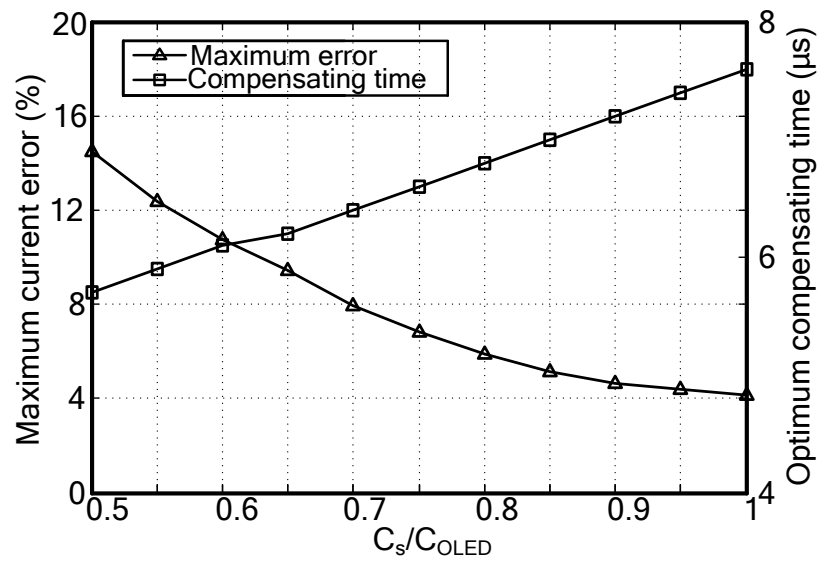

Fig. 10: Profile of the optimum compensation time and maximum current error as a function of $\alpha=C_{s} / C_{\mathrm{OLED}}$.

one technology (as it is) and apply it to the circuit implemented in the other technology, it will work, probably with a different rate of error. In order to adjust the error to its minimum, we need to make some modifications in both the circuit parameters and the timings of the waveform itself. What we should do first is to choose proper sizes for the TFTs. As already mentioned, we choose the same size for all the constituent components of the two circuit implementations in the two technologies. As for the drive TFT, the aspect ratio is chosen to provide enough driving current for the OLED. This sets the lower limit. The upper size limit, however, is imposed by any inaccuracy in the time setting during the compensation phase. A timing error can occur because of the driving circuits themselves or as a result of different interconnect-associated delays of different pixels over the display area. Assuming a maximum timing error of $\Delta t$, the voltage deviation during the compensation phase would be

$$
\Delta V_{c}=\frac{K}{C_{s}} V_{\mathrm{ov}}^{2} \Delta t .
$$

Thus, a large $\mathrm{K}$ would be followed with a large voltage error. The same conclusion can be drawn with regard to $V_{\mathrm{ov}}$ during the compensation phase. This too, puts a limit on choosing
TABLE I: Parameters of the circuit.

\begin{tabular}{|c|c|c|}
\hline & \multicolumn{2}{|c|}{ Value } \\
\hline Parameter & a-Si & a-IGZO \\
\hline$T_{1}$ & $\mathrm{~W} / \mathrm{L}=10 \mu \mathrm{m} / 10 \mu \mathrm{m}$ & $\mathrm{W} / \mathrm{L}=10 \mu \mathrm{m} / 10 \mu \mathrm{m}$ \\
\hline$S_{1}$ & $\mathrm{~W} / \mathrm{L}=20 \mu \mathrm{m} / 10 \mu \mathrm{m}$ & $\mathrm{W} / \mathrm{L}=20 \mu \mathrm{m} / 10 \mu \mathrm{m}$ \\
\hline$S_{2}$ & $\mathrm{~W} / \mathrm{L}=20 \mu \mathrm{m} / 10 \mu \mathrm{m}$ & $\mathrm{W} / \mathrm{L}=20 \mu \mathrm{m} / 10 \mu \mathrm{m}$ \\
\hline$C_{s}$ & $1 \mathrm{pF}$ & $1 \mathrm{pF}$ \\
\hline$V_{\text {data }}$ & $0 \mathrm{~V} \sim 20 \mathrm{~V}$ & $0 \mathrm{~V} \sim 13 \mathrm{~V}$ \\
\hline scan1 & $0 \mathrm{~V} / 30 \mathrm{~V}$ & $0 \mathrm{~V} / 20 \mathrm{~V}$ \\
\hline scan2 & $0 \mathrm{~V} / 30 \mathrm{~V}$ & $0 \mathrm{~V} / 20 \mathrm{~V}$ \\
\hline$V_{\text {DD }}$ & $0 \mathrm{~V} / 10 \mathrm{~V} / 20 \mathrm{~V}$ & $0 \mathrm{~V} / 20 \mathrm{~V}$ \\
\hline$V_{\mathrm{SS}}$ & $0 \mathrm{~V} / 30 \mathrm{~V}$ & $0 \mathrm{~V} / 20 \mathrm{~V}$ \\
\hline
\end{tabular}

TABLE II: Summery of model parameters.

\begin{tabular}{|c|c|c|}
\hline & \multicolumn{2}{|c|}{ Value } \\
\hline Parameter & $\mathrm{a}-\mathrm{Si}$ & $\mathrm{a}-\mathrm{IGZO}$ \\
\hline Equivalent field effect mobility $\left(\mu_{\mathrm{FET}}\right)$ & $1.2 \mathrm{~cm}^{2} V^{-1} s^{-1}$ & $15 \mathrm{~cm}^{2} V^{-1} s^{-1}$ \\
\hline Sub-threshold slope $\left(S_{f 0}\right)$ & $0.5 \mathrm{~V} / \mathrm{dec}$ & $0.25 \mathrm{~V} / \mathrm{dec}$ \\
\hline Reference OFF current $\left(I_{\text {offo }}\right)$ & $5 \mathrm{pA}$ & $1 \mathrm{pA}$ \\
\hline Normalized contact resistance $\left(R_{c} W\right)$ & $1000 \Omega-\mathrm{cm}$ & $100 \Omega-\mathrm{cm}$ \\
\hline Threshold voltage $\left(V_{\text {th }}\right)$ & $2 \mathrm{~V}$ & $2 \mathrm{~V}$ \\
\hline Normalized overlap capacitance & $8 \mathrm{pF} / \mathrm{cm}$ & $8 \mathrm{pF} / \mathrm{cm}$ \\
\hline Normalized gate insulator capacitance & $20 \mathrm{nF} / \mathrm{cm}^{2}$ & $20 \mathrm{nF} / \mathrm{cm}^{2}$ \\
\hline
\end{tabular}

an appropriate value for $V_{\text {data2 }}$, which is in trade-off with the maximum voltage error in (16).

For the switches, the limited size of the pixel does not allow us to incorporate large sizes to have better conductivity and higher speed. The pedestal error (clock feedthrough and charge injection) is another factor that needs to be taken into consideration in choosing the switch size. Due to very low ON resistance of the a-Si transistors, a higher driving voltage of $30 \mathrm{~V}$ is chosen to have a reasonable settling time. Apart from that, since the maximum data voltage for a-Si implementation is $20 \mathrm{~V}$ (to have the same maximum OLED current as the aIGZO implementation), the gate voltage of switch $S_{2}$ must be sufficiently larger than $20 \mathrm{~V}$ to allow a low enough switch resistance.

Another discrepancy between the two technologies arises during the forth phase when $V_{\mathrm{DD}}$ needs to drop to a lower voltage. As can be seen in Fig. (7), after the third phase, $V_{\mathrm{DD}}$ is pulled down to a mid-level and not to the ground. Otherwise, the final voltage stored on $C_{s}$, which then provides the overdrive biasing voltage of $T_{1}$, would be too small that cannot supply a reasonably large enough current to drive the OLED. Due to almost one order of magnitude higher mobility of an a-IGZO TFT, this need not be done for the other circuit, thus giving us the advantage of employing a two-level supply voltage rather than a three-level one.

\section{Simulation Results}

The proposed pixel circuit with parameters listed in Table I has been implemented with both a-Si and a-IGZO TFTs, and simulated with Verilog-AMS extracted model based on real measurement data [13-16]. A list of important parameters used in the models is summarized in Table II.

A sample transient waveform of the drain and gate voltage of the drive a-Si TFT is illustrated in Fig. 11. The waveforms

\footnotetext{
${ }^{5} \mathrm{An}$ overlap of $1 \mu \mathrm{m}$ on drain and source sides for the minimum gate length of $10 \mu \mathrm{m}$ is assumed.
} 


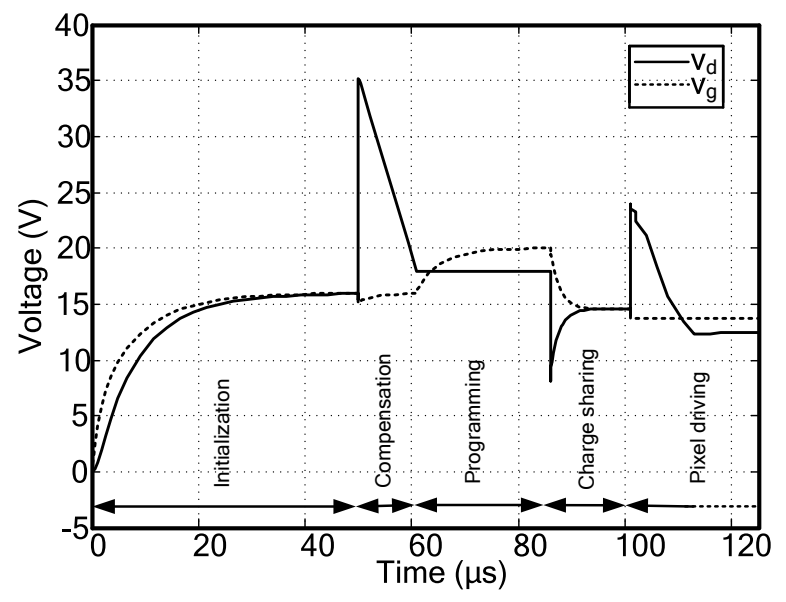

Fig. 11: Transient waveform of gate and drain voltages using a-Si TFTs.

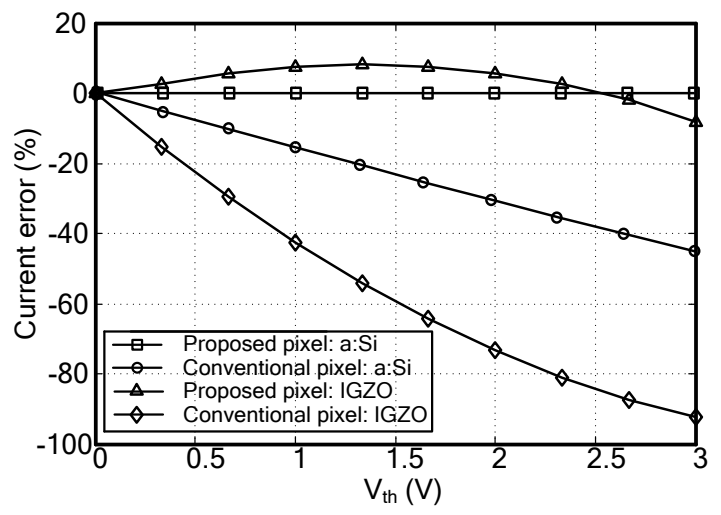

Fig. 12: Measured current error as a function of the threshold voltage shift in the drive TFT.

are similar for a-IGZO model. The total programming times are $91 \mu s$ and $26 \mu s$ for the a-Si and the a-IGZO implementation, respectively. Fig. 12 shows the current error for a $3 \mathrm{~V}$ shift in the threshold voltage of $T_{1}$ as well as the relative error in a conventional 2-TFT pixel (Fig. (1)) with the same size of the drive and the switch TFTs used in the proposed circuit. As can be seen, the maximum error is almost zero for the a-Si circuit, while this is around $8 \%$ for the a-IGZO implementation. The larger error of a-IGZO circuit is because of the low value of overdrive voltage during the compensation phase $\left(V_{\mathrm{ov}}=5 V\right)$. A larger value, as explained in section III(B), would result in high susceptibility to any timing error of the pixel. The overdrive voltage for a-Si circuit during the compensation period is $14 \mathrm{~V}$. The profiles of the OLED current versus $V_{\text {data }}$ are also depicted in Fig. 13 and Fig. 14.

\section{CONCLUSION}

A pixel circuit comprising three TFTs and one capacitor (3T1C), controlled by two scan lines is presented. For maintain fair comparison, identical component sizes is chosen for the two circuits in two different technologies of the same pixel area. The simulation results using established VerilogA models show that the maximum non-uniformity in the OLED current

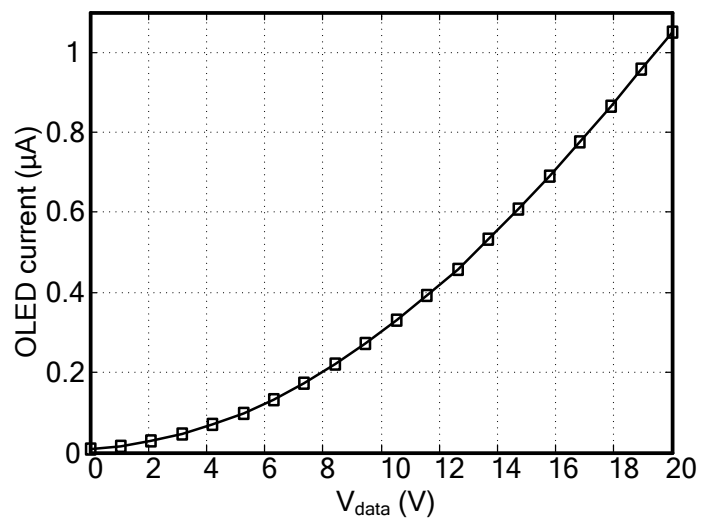

Fig. 13: OLED current as a function of $V_{\text {data }}$ (a-Si implementation).

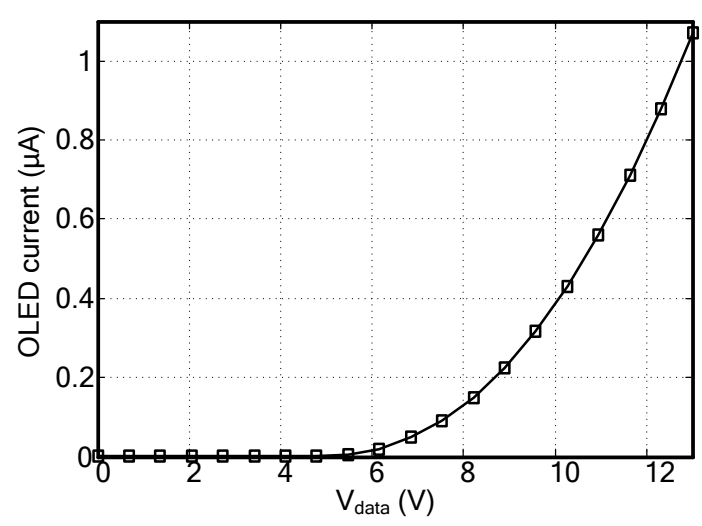

Fig. 14: OLED current as a function of $V_{\text {data }}$ (a-IGZO implementation).

is near zero for the a-Si implementation when experiencing a $3 \mathrm{~V}$ shif of the threshold voltage. The a-IGZO circuit, however, shows an error of around $8 \%$ while being 3.5 times faster than its equivalent a-Si circuit. This demonstrates that the accuracyspeed trade-off of transistor-based circuits holds here as well.

Compared to the 2-TFT pixel, the circuit presented here requires an additional TFT and scan line. As such, the circuit would not impose restrictions on pixel size, although the requirements on driving sequence including switching power lines may require a custom driver. The pixel layout needs to be optimized so as to accommodate the size of the OLED capacitor, which is crucial in determining the compensation time.

\section{ACKNOWLEDGEMENT}

Authors thank to the EPSRC under Project EP/M013650/1.

\section{REFERENCES}

[1] G.R. Chaji and A. Nathan, " LTPS vs Oxide Backplanes for AMOLED Displays: System Design Considerations and Compensation Techniques", SID Symposium Digest of Technical Papers, Vol. 45, Issue 1, pp. 153-156, 2014.

[2] K. Nomura, H. Ohta, A. Takagi, T. Kamiya, M. Hirano, and H. Hosono, "Room-temperature fabrication of transparent flexible thin-film transistors using amorphous oxide semiconductors", Nature, vol. 432, pp. 488-492, 2004 
[3] J. Jang, M. Mativenga and J. Choi, "Stabilities of TFTs under bias-stress", ECS Transactions, vol. 33, pp. 31-39, 2010.

[4] S. Jeon, S. Ahn, I. Song, C. Kim, U. Chung, E. Lee, I. Yoo, A. Nathan, S. Lee, K. Ghaffarzadeh, J. Robertson, and K. Kim, "Gated threeterminal device architecture to eliminate persistent photoconductivity in oxide semiconductor photosensor arrays", IEEE Journal of Solid-State. Circuits, vol. 39, no. 9, pp. 1477-1486, 2004.

[5] A. Nathan, A. Kumar, K. Sakariya, P. Servati, S. Sambandan, and D. Striakhilev, "Amorphous silicon thin film transistor circuit integration for organic LED displays on glass and plastic", IEEE Journal of SolidState. Circuits, vol. 39, no. 9, pp. 1477-1486, 2004.

[6] J. Goh, H-J. Chung, and J. Jang, "A new pixel circuit for active matrix organic light emitting diodes", IEEE Electron Device. Lett., vol. 23, no. 9, pp. 544-546, 2002.

[7] J. Goh, J. Jang, K. Cho, and C. Kim, "A new a-Si:H thin-film transistor pixel circuits for active matrix organic light-emitting diodes", IEEE Electron Device. Lett., vol. 24, no. 9, pp. 583-585, 2003.

[8] G.R. Chaji, P. Servati, and A. Nathan, "driving scheme for stable operation of the 2-TFT a-Si AMOLED pixel", Electron. Lett., vol. 41, pp. 499500, Apr. 2005.

[9] B-H. You, J-H. Lee, and M-K. Han, "Polarity Balanced driving scheme to suppress the degradation of Vth in a-Si:H TFT due to the positive gate bias stress for AMOLED", IEEE Journal of Display Technology, vol. 3, no. 2, pp. 40-44, 2007.

[10] A. Nathan, R. Chaji, and S-J. Ashtiani, "Driving Schemes for a-Si and LTPS AMOLED displays", IEEE/OSA Journal of Display Technology, vol. 1 , no. 2, pp. 267-277, 2005.

[11] M. Bagheri, S. J. Ashtiani, and A. Nathan, "A Fast Driving Scheme Voltage-Programmed Pixel Structure for AMOLED Displays", IEEE Journal of Display Technology, vol. 6, pp. 191-195, Apr. 2010.

[12] J. Jacobs, D. Hente, E. Waffenschmidt, "Driving for OLEDs", IEEE 42nd Industry Applications Conference. 200. pp. 1147-1152, Sept. 2007.

[13] P. Servati, D. Striakhilev, and A. Nathan, "Above-threshold parameter extraction and modeling for amorphous silicon thin-film transistors", IEEE Trans. Electron Devices, vol. 50, no. 11, pp. 2227-2235, 2003.

[14] S. Lee, A. Nathan et al., "Modeling Sub-Threshold Current-Voltage Characteristics in Thin Film Transistors", IEEE Journal of Display Technology, vol. 9, no.11, Nov. 2013.

[15] S. Lee, A. Nathan et al., "LateNews Paper: Modeling CurrentVoltage Behaviour in Oxide TFTs Combining Traplimited Conduction with Percolation", Digest of Technical papers, International Symposium, Display Week 2013, SID 2013, vol. 44, no. 1, pp. 2225, June 2013.

[16] S. Lee, A. Nathan et al., "Analytical Field-Effect Method for Sub-gap States Extraction in Amorphous Semiconductor Thin Film Transistors", IEEE Electron Device Letters, vol.33, no.7, Jun. 2012.

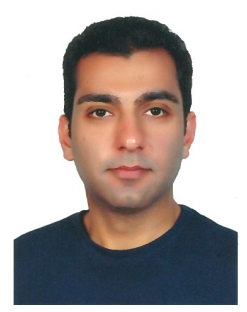

Mojtaba Bagheri received the B.S. degree from the University of Tehran, Iran and the M.S degree (magna cum laude) in Electronics and Integrated Circuits from the KU Leuven, Belgium. For his Master's thesis he was focusing on designing veryhigh speed Flash analog-to-digital converters in standard CMOS technology. He worked as an intern at nSilition for one year, where he was involved in designing analog IPs in advanced CMOS technologies. At present, he is a $\mathrm{PhD}$ student at the University of Cambridge, working on design of high performance A/D converters as well as integrated analog circuits for wearable electronics and communication systems in both CMOS and flexible thin film technologies.

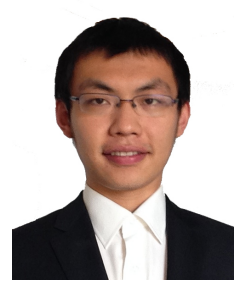

Xiang Cheng received his bachelor degree in electronic engineering from Shanghai Jiao Tong University in 2013. During the degree, he worked on AMOLED pixel circuit design and simulation. Currently, he is a Ph.D student in University of Cambridge. His current interests include biosensors, circuit design and TFT systems.

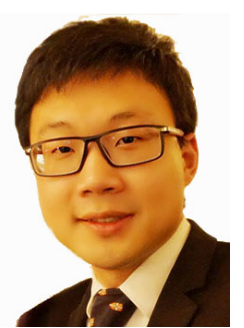

Junhao Zhang is currently working toward the $\mathrm{PhD}$ degree in Unversity of Cambridge. Before joining Cambridge, he was at the Institute of Chemistry (CAS), National Center for Nanoscience \& Technology, National Taiwan University and USTB. He has also worked in ARM on chip design based on $14 \mathrm{~nm}$ and $16 \mathrm{~nm}$ FinFET technologies.

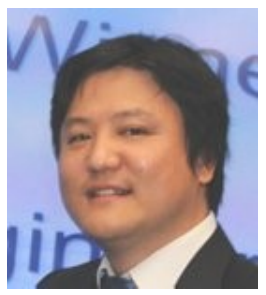

Sungsik Lee is currently a research associate in the Department of Engineering at University of Cambridge. He received his $\mathrm{PhD}$ degree from University College London (UCL) where he worked on physics and modelling of amorphous oxide thin film transistors. His area of expertise is an investigation of physics and modelling of advanced semiconductor devices for designing futuristic circuits and systems.He has been an authored/co-authored over 60 articles and 9 US patents. He won various awards including the IEEE EDS PhD Fellowship 2011 and ABTA doctoral researcher award 2013. He is a technical program committee member of international conferences (IEEE AP Sensors / ISSII / Sensors), and a guest editor of IEEE JDT. He is a member of IEEE and IEEE EDS.

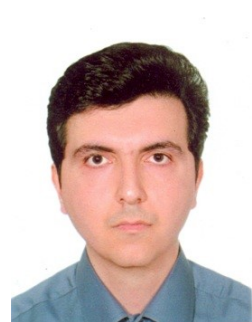

Shahin J. Ashtiani received the B.Sc. (Hons.) and M.A.Sc (Hons.) degrees from the University Of Tehran, Iran in 1998 and 2001, respectively in electronics engineering. He received his $\mathrm{PhD}$ degree from University of Waterloo, Waterloo, Ontario, Canada in 2007. His PhD project was on driver circuits for active-matrix organic light-emitting diode (AMOLED) displays. From 2001 to 2003 he was with Valence Semiconductor Inc. as an analog design engineer, working on low-power, high-speed analogto-digital converters. Since 2008, his is an assistant professor at the school of electrical and computer engineering, University of Tehran, Iran. His current research interests are large-area electronics, lab-onchip, biomedical instrumentation, and wireless power and data transfer.

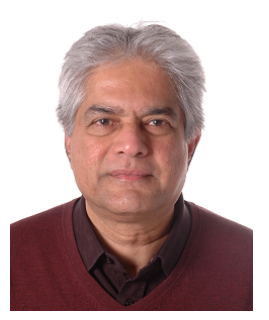

Arokia Nathan Prof. Arokia Nathan holds the chair for Photonic Systems and Displays in the Department of Engineering. His research addresses the heterogeneous system integration with emerging materials for large area flexible electronics for human-machine interfaces and wearable devices. He is cofounder of four spin-off companies, including Ignis Innovation Inc. He received the Royal Society Wolfson Research Merit Award in 2006 and is a Fellow of IEEE (USA) and the IET (UK), and an IEEE/EDS Distinguished Lecturer. He has published over 500 papers in the field of sensor and thin film electronics technology and $\mathrm{CAD}$ and is an author/co-author on more than 50 patents filed/awarded. 